\title{
Capturing the spatiotemporal dynamics of self-generated, task-initiated thoughts with EEG and fMRI
}

\author{
Lucie Bréchet $^{\mathrm{a}, \mathrm{b}}$, Denis Brunet ${ }^{\mathrm{b}, \mathrm{c}}$, Gwénaël Birot ${ }^{\mathrm{d}}$, Rolf Gruetter ${ }^{\mathrm{a}, \mathrm{c}}$, Christoph M. Michel ${ }^{\mathrm{b}, \mathrm{c}, *}$, \\ João Jorge ${ }^{\mathrm{a}}$ \\ ${ }^{a}$ Laboratory for Functional and Metabolic Imaging, EPFL, Lausanne, Switzerland \\ ${ }^{\mathrm{b}}$ Functional Brain Mapping Laboratory, Fundamental Neuroscience Dept., University Geneva, Switzerland \\ ${ }^{\mathrm{c}}$ Biomedical Imaging Research Center (CIBM), Lausanne, Geneva, Switzerland \\ ${ }^{\mathrm{d}}$ EEG-BCI Facility, Fondation Campus Biotech Geneva, Switzerland
}

\begin{abstract}
A B S T R A C T
The temporal structure of self-generated cognition is a key attribute to the formation of a meaningful stream of consciousness. When at rest, our mind wanders from thought to thought in distinct mental states. Despite the marked importance of ongoing mental processes, it is challenging to capture and relate these states to specific cognitive contents. In this work, we employed ultra-high field functional magnetic resonance imaging (fMRI) and high-density electroencephalography (EEG) to study the ongoing thoughts of participants instructed to retrieve self-relevant past episodes for periods of 22sec. These task-initiated, participant-driven activity patterns were compared to a distinct condition where participants performed serial mental arithmetic operations, thereby shifting from self-related to self-unrelated thoughts. BOLD activity mapping revealed selective enhanced activity in temporal, parietal and occipital areas during the memory compared to the mental arithmetic condition, evincing their role in integrating the re-experienced past events into conscious representations during memory retrieval. Functional connectivity analysis showed that these regions were organized in two major subparts, previously associated to "scene-reconstruction" and "self-experience" subsystems. EEG microstate analysis allowed studying these participant-driven thoughts in the millisecond range by determining the temporal dynamics of brief periods of stable scalp potential fields. This analysis revealed selective modulation of occurrence and duration of specific microstates in the memory and in the mental arithmetic condition, respectively. EEG source analysis revealed similar spatial distributions of the sources of these microstates and the regions identified with fMRI. These findings imply a functional link between BOLD activity changes in regions related to a certain mental activity and the temporal dynamics of mentation, and support growing evidence that specific fMRI networks can be captured with EEG as repeatedly occurring brief periods of integrated coherent neuronal activity, lasting only fractions of seconds.
\end{abstract}

\section{Introduction}

Spontaneous mentation is neither random nor meaningless (Smallwood and Schooler, 2015), however how to precisely capture the wandering mind and attribute it to specific cognitive thoughts, is yet unclear (Fox et al., 2015). Functional magnetic resonance imaging (fMRI) as well as electroencephalography (EEG) studies have previously related resting-state activity to cognitive processes mainly indirectly, by using post-scan questionnaires (Andrews-Hanna et al., 2010b; Fox et al., 2018; Kucyi, 2018) or by asking participants about their ongoing thoughts during the spontaneous mentation (Kucyi et al., 2016; Perogamvros et al., 2017; Stawarczyk and D'Argembeau, 2015). The striking similarities found by comparing the spatial distributions of large-scale networks specific to self-reported thoughts and those initiated by experimental tasks (Andrews-Hanna et al., 2010b; Axelrod et al., 2017; Delamillieure et al., 2010; Shirer et al., 2012), contribute to the wide debate about the content of spontaneous mental activity at rest and the relation of resting state networks (RSN) to cognitive networks (Biswal et al., 1995; Christoff et al., 2016; Davis et al., 2017; Kucyi et al., 2016; Power et al., 2011; Raichle, 2015; Smith et al., 2009; Spreng and Schacter, 2012).

Time-resolved fMRI studies previously demonstrated that the RSNs spontaneously fluctuate in and out of spatially and temporally overlapping correlations (Cabral et al., 2017; Cole et al., 2014; Cole et al., 2013; de Pasquale et al., 2017; Hutchinson and Raff, 2014; Karahanoglu and Van De Ville, 2015; Power et al., 2014; Vidaurre et al., 2017; Zalesky et al., 2014), indicating that temporal dynamics of resting state activity are not stationary, but rather partitioned into distinct epochs. This observation corroborates the prevailing concept that spontaneous mental activity is discontinuous and parsed into a series of conscious states that manifest discrete spatiotemporal patterns of neuronal activity (for review see (Deco et al., 2011; Meehan and Bressler, 2012; Michel and Koenig, 2018)). However, despite much progress in dynamic resting-state functional neuroimaging, the delayed and slow hemodynamic response to neuronal activity limits fMRI methods to capture these states (Vidaurre

\footnotetext{
* Corresponding author. Functional Brain Mapping Laboratory, Fundamental Neuroscience Dept., University Geneva, Switzerland.

E-mail address: christoph.michel@unige.ch (C.M. Michel).
} 
et al., 2017). Indeed, to efficiently execute mental processes, large-scale networks have to dynamically re-organize on sub-second temporal scales (Bressler and Menon, 2010; de Pasquale et al., 2017).

EEG microstate analysis allows to investigate these fast temporal dynamics of large-scale neural networks and to access information about the functional organization of spontaneous mentation in time (Koenig et al., 2002; Lehmann, 1990; Michel and Koenig, 2018; van de Ville et al., 2010). EEG microstates reflect brief epochs of coherent neuronal activity that persist for around $100 \mathrm{~ms}$ (Khanna et al., 2015; Michel and Koenig, 2018). Previous studies demonstrated that the occurrence, duration and sequence of EEG microstates determine the quality of spontaneous mentation, and as such could represent the basic building blocks of conscious mental processes (Lehmann, 1990). EEG microstates thus qualify as the electrophysiological manifestation of the segmentation of ongoing mental activity into short-lasting brain states (Baars, 2002a; Changeux and Michel, 2004; Michel and Koenig, 2018). Only a few studies have tried to relate EEG microstates to specific cognitive processes (Lehmann et al., 1998; Milz et al., 2016a; Seitzman et al., 2017) and none of these studies compared the EEG microstates directly to the underlying brain networks observed with fMRI during the same processes.

Recent studies have explored novel experimental designs based on task-initiated spontaneous activity, whereby participants were asked to think freely, but with specific instructions on the thoughts they should focus on (Andrews-Hanna et al., 2010b; Axelrod et al., 2017; Delamillieure et al., 2010). For example, a recent fMRI study by Axelrod and colleagues (Axelrod et al., 2017) explored the task-initiated self-generated processing to distinguish different brain processes that are at work during internal mentation. This study showed that the Default Mode Network (DMN) dominated during self-generated processing, independent of the type of mental experience (future/past imagery, episodic memory, empathizing). The authors then compared this activation pattern with the brain responses to specific cognitive tasks, i.e. self-referential processing, scene reconstruction and language-related processing, and showed that different parts of the DMN correlated with these task-related networks. These observations thus demonstrated that different distinct cognitive processes are activated during internal mentation.

The overall goal of this study was to capture the occurrence of these different cognitive processes in time using ultra-high field 7T fMRI and high-density 64-channel EEG recordings to obtain both hemodynamic and electrophysiological signatures with high spatial and temporal sensitivity. The use of ultra-high field fMRI was particularly sought to boost the trade-off between detection sensitivity and spatial specificity, particularly important to capture differences in activity across spatiallyclose brain regions, such as sub-areas of the DMN (Foster et al., 2015). Fifteen healthy participants were recorded during spontaneous mentation (hereafter termed "rest condition") and while focusing their thoughts repeatedly for periods of 22sec with eyes closed on either episodic, self-related memories associated with a briefly presented image ("memory condition"), or arithmetic calculations ("math condition"). Consistent with cognitive tasks that require working memory and direct the attention outside of the self (Corbetta and Shulman, 2002), we chose the math condition as a control condition in order to selectively de-activate regions that become active in autobiographic episodic memory retrieval (Foster et al., 2012, 2015).

The experimental design was conceived so that the recording sessions were dominated by the periods of undisturbed, subject-driven mentation, yet remaining within the same known cognitive condition (memory, math, or rest) for several minutes. This enabled the use of techniques more typically akin to resting-state fMRI studies, allowing us to study condition-specific changes in both BOLD activity and connectivity, in multiple specific brain regions and their organization in large-scale networks. Simultaneously, this experimental design was also well suited for EEG microstate analysis (Michel and Koenig, 2018), allowing us to examine the fast temporal organization of the brain's large-scale network dynamics. Specifically, we focused on the most basic characteristics of the EEG microstate temporal dynamics: their duration, their frequency of occurrence and their transition probabilities. Finally, we estimated the brain networks generating the EEG microstates and compared them to the fMRI networks.

\section{Methods}

\subsection{Participants and experimental paradigm}

We included 15 healthy participants $(30.5 \pm 5.5 y e a r s, 5$ male $/ 10$ female) in this study. The work was approved by the institutional review board of the local ethics committee, and all participants provided written informed consent prior to the experiment.

Each participant underwent three sessions: i. an interview session, ii. an EEG recording session, and iii. an fMRI session. During the interview, all participants performed a classical autobiographical memory questionnaire (ABMQ) (Rubin et al., 2003) in order to select vividly remembered images, which were then used for the EEG and for the fMRI sessions, which included three distinct conditions each (Fig. 1): eyes-closed rest $(6 \mathrm{~min})$, mentally retrieving personal past episodes (10min), and mental arithmetic operations (10min). The memory and math conditions comprised twenty 30-s trials each. On each trial, a personal image (e.g., photo of a participant with a birthday cake) or a calculation (e.g., $447-7=$ ) was presented for $2 \mathrm{sec}$, and then faded to black over an additional $1 \mathrm{~s}$. This was followed by a $22 \mathrm{sec}$ period of closed-eyes during which the participants retrieved the past event or continued to serially subtract/add the given number. After this period, subjects were alerted by a light flash to open their eyes and a question was presented on the screen: "How much did you relive the original event?" for the memory task and "How much attention did you pay to the calculation?" for the mental arithmetic task. The time allowed to read and respond to the question was $5 \mathrm{sec}$. Participants answered by a button press ( $1=$ "not at all", to $4=$ "fully"). The ratings confirmed active involvement of our participants with high confidence ratings that were comparable between the EEG $(M=3.2, S E M=0.12)$ and the fMRI $(M=3.1, S E M=0.11)$ memory session as well as the EEG $(M=3.5$, $\mathrm{SEM}=0.12)$ and the fMRI $(\mathrm{M}=3.41, \mathrm{SEM}=0.11)$ math session.

Within this experimental design, the periods of undisturbed, subjectdriven eyes-closed mentation composed the large majority of the paradigm duration (roughly $73 \%$, i.e. 22 s out of every 30 s). The external cues (pictures or arithmetic expressions) were merely intended to guide and maintain the mind wandering within the same cognitive context, and were therefore kept as brief as possible.

Each experimental session started with a 6 min eyes-closed rest condition followed by two counter-balanced experimental conditions. In the memory conditions, participants saw a personal image for $3 \mathrm{sec}$ (2sec static, then $1 \mathrm{sec}$ fading to black), after which they closed their eyes and had to retrieve the event. After each trial, participants were prompted by a brief flash of light to open their eyes, and answered a control question with a button press: "How much did you relive the original event?" (scale $1=$ "not at all" to $4=$ "fully"). Before the scanning, all participants performed a classical autobiographical memory questionnaire (ABMQ) (Rubin et al., 2003) in order to select the 20 most vividly remembered images that were then shown during the task. In the math condition, participants saw a formula presented for 3 sec on the screen. They then closed their eyes and proceeded to continually subtract the number from the preceding result for 22sec. After each trial, participants answered to the question "How much attention did you pay to the calculation?" by button press (scale $1=$ "not at all" to $4=$ "fully").

\section{2. fMRI acquisition and analysis}

\subsection{1. fMRI acquisition}

The fMRI sessions were conducted on an actively shielded Magnetom 7T head scanner (Siemens, Erlangen, Germany), equipped with AC84 


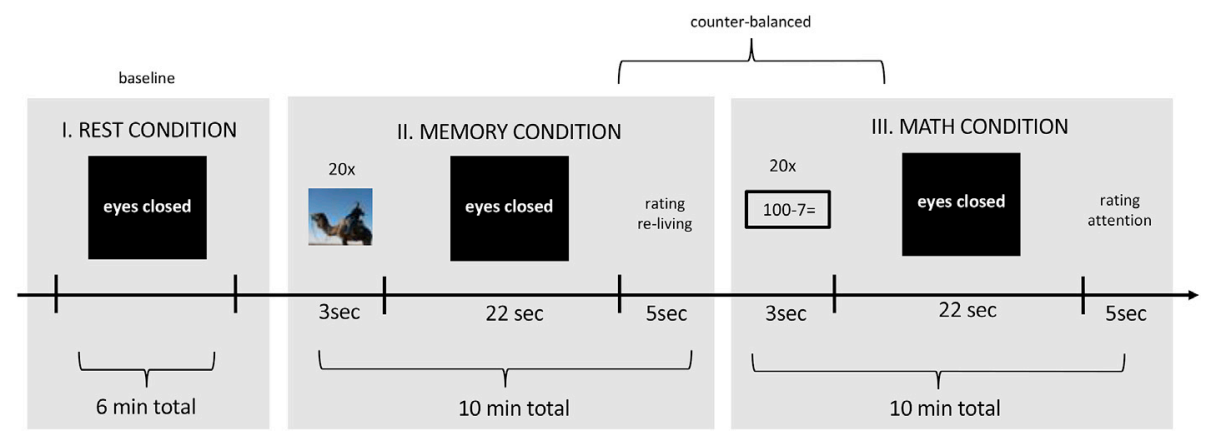

Fig. 1. Experimental paradigm.

head gradients $(80 \mathrm{mT} / \mathrm{m}$ max. gradient strength, $333 \mathrm{~T} / \mathrm{m} / \mathrm{s}$ max. slewrate) and an 8-channel transmit/receive head loop array (Rapid Biomedical, Rimpar, Germany). During each paradigm, whole-brain functional data were acquired using a simultaneous multi-slice (SMS) 2D GE-EPI sequence, with TR/TE $=1000 / 22 \mathrm{~ms}, \alpha=54^{\circ}, 110 \times 110$ matrix size with $2.0 \mathrm{~mm}$ isotropic spatial resolution, 75 sagittal slices with $3 \times$ SMS acceleration and 1/3 field-of-view (FOV) CAIPI shift (Setsompop et al., 2012), $2 \times$ in-plane GRAPPA acceleration (anterior-posterior direction) and $7 / 8$ partial Fourier undersampling. This protocol was designed to simultaneously provide fairly high temporal resolution $(1 \mathrm{vol} / \mathrm{s})$ and spatial resolution (2-mm voxel width), with whole-brain coverage. For each subject, an additional 5-vol scan was also performed with reversed phase encoding direction (posterior-anterior), for subsequent correction of susceptibility-induced EPI distortions. To aid spatial co-registration, $\mathrm{T}_{1}$-weighted anatomical data were acquired with a 3D MP2RAGE sequence (Marques et al., 2010) with TR/TI 1 /$\mathrm{TI}_{2} / \mathrm{TE}=5500 / 750 / 2350 / 1.87 \mathrm{~ms}$ and $1.0 \mathrm{~mm}$ isotropic spatial resolution.

\subsection{2. fMRI pre-processing}

The fMRI data were processed using the FMRIB Software Library (FSL v5.0, Oxford, UK) combined with Matlab routines developed in-house (Mathworks, Natick MA, USA). Data pre-processing included motion correction (MCFLIRT tool, 6 degrees of freedom) (Jenkinson et al., 2012), slice-timing adjustment (set to the middle of each TR, via linear interpolation), brain segmentation (BET tool) (Smith, 2002), Gaussian spatial smoothing (FWHM $=4 \mathrm{~mm}$ ) and temporal de-trending (100sec cut-off). To reduce contributions from head motion, a set of 24 confound regressors derived from the motion parameter time courses were regressed out of the fMRI data by general linear model analysis. Each dataset was co-registered to the standard MNI space as follows: first, for each subject, the fMRI data were $\mathrm{B}_{0}$-unwarped using FSL-TOPUP (Andersson et al., 2003) and brought to the subject's anatomical space using FLIRT with boundary-based registration (Greve and Fischl, 2009) (12 degrees of freedom). The co-registration to MNI space was then determined using the anatomical images, again through FLIRT (12 degrees of freedom).

\subsubsection{Fractional amplitude of low frequency fluctuations (fALFF) analysis}

The prevailing dominance of periods of undisturbed, eye-closed subject-driven mentation over the recording sessions (only interrupted by brief external cues), motivated the use of techniques more typically used in resting-state fMRI studies, and enabled the study of both BOLD activity and connectivity with such techniques. Accordingly, voxel-wise fALFF values were estimated for the pre-processed fMRI data of each subject and paradigm, in native space, as proposed by Zou et al. (2008): the time series of each voxel was transformed to the frequency domain and the sum of amplitudes in the $0.01-0.08 \mathrm{~Hz}$ interval was divided by the sum of amplitudes of the full frequency band. The individual fALFF maps thus obtained for each paradigm and subject were then brought to MNI space for group analysis. The fALFF maps were compared between the memory and math conditions using paired T-tests across subjects, and statistical significance was determined using topological false-discovery rate (FDR) inference to correct for multiple comparisons (Chumbley et al., 2010) (cluster-forming threshold $\mathrm{T}>2.20$, FDR $\alpha=5 \%$ ). This topological FDR approach has demonstrated excellent sensitivity in previous studies at 7T conducted by our group, even with smaller voxel size and lower levels of smoothing (Jorge et al., 2018).

After statistical testing, the resulting clusters were further analysed to determine whether the significant differences observed between memory and math were due to increases and/or decreases in BOLD activity for each of those conditions, relative to baseline. To this end, for each subject and brain voxel, the difference in fALFF between the memory (or math) and the rest condition was computed, and then averaged across subjects and voxels within each of the cluster ROIs.

\subsubsection{Functional connectivity analysis}

The brain regions (clusters) displaying significant fALFF changes between the memory and math conditions were also further considered for connectivity analysis. These regions were warped to each individual run's native space, and the voxels belonging to each region were identified. The functional connectivity between each pair of regions was estimated as the average Pearson correlation value between the full time course of every voxel in the first region with that of every voxel in the second region. The estimations were organized in a connectivity matrix, and also included an estimation for each region paired with itself, serving as a measure of functional homogeneity within the region.

\subsection{EEG acquisition and analysis}

\subsubsection{EEG recording and pre-processing}

EEG was recorded with a 64-channel BrainAmp EEG system (Brain Products, Munich, Germany) at $5000 \mathrm{~Hz}$. Offline, the EEG was downsampled to $250 \mathrm{~Hz}$ and band-pass filtered between $1 \mathrm{~Hz}$ and $40 \mathrm{~Hz}$ using a noncausal filter (2nd order butterworth Low and High pass, - 12 $\mathrm{db} /$ octave roll-off, computed linearly forward and backward, eliminating the phase shift, and with poles calculated each time to the desired cut-off frequency). Infomax-based Independent Component Analysis (ICA) was applied to remove oculomotor and cardiac artefacts based on the channels with maximal amplitude, the topography, and time course of the ICA component. Bad electrodes were then interpolated using a 3-D spherical spline (Perrin et al., 1989) and the data was re-computed to the common average-reference. For the memory and the math condition, all $20 \mathrm{~s}$ epochs of eyes closed mentation, starting $5 \mathrm{~s}$ after stimulus presentation and ending with the flash alert to open the eyes, were concatenated. Finally, the data were visually inspected and periods with remaining artefacts were marked and excluded from further analysis.

\subsubsection{K-means clustering}

The free academic software Cartool was used for the microstate analysis (Brunet et al., 2011). A modified k-means cluster analysis (Pascual-Marqui et al., 1995) was applied to the data of each subject and condition. Only maps at local maxima of the Global Field Power (GFP) 
entered the cluster analysis, as they represent time points of highest signal-to-noise ratio (Koenig and Brandeis, 2016; Koenig et al., 2002). Polarity of the maps was ignored in the clustering. Cluster-analysis was applied in two steps: first on the data of each individual subject and condition, and then on the cluster maps derived from each subject within a condition. In order to determine the optimal number of clusters (both within and across subjects), 7 criteria were used to evaluate independently the quality of each clustering. They were then merged together in order to derive a single synthetic meta-criterion (Custo et al., 2017). This improves confidence in the right estimation of the optimal number of clusters, as compared to previous work relying on a single criterion only (i.e. Cross-Validation criterion (Pascual-Marqui et al., 1995) or the Krzanowski-Lai Index (Murray et al., 2008)).

The following 7 criteria were taken from (Charrad et al., 2014; Krzanowski and Lai, 1988; Milligan and Cooper, 1985; Pascual-Marqui et al., 1995):

1. Gamma: An adaptation of Goodman and Kruskal, based on concordant vs. discordant clustered pairs.

2. Silhouettes: Evaluation of the consistency of each cluster through its goodness of fit.

3. Davies and Bouldin: A function of the sum of the ratio of within-cluster to between-cluster separation.

4. Point-Biserial: A point-biserial correlation calculated between the distance matrix and a binary cluster index.

5. Dunn: An evaluation of the goodness of separation of all clusters.

6. Krzanowski-Lai Index: A ratio of the relative difference of the withinclusters dispersion.

7. Cross-Validation: A modified version of the predictive residual variance.

The meta-criterion was defined as the median of all optimal numbers of clusters across all criteria. The meta-criterion calculation is implemented in the free academic software Cartool (https://sites.google.com/ site/cartoolcommunity/).

\subsubsection{Back-fitting}

The group cluster maps of a given condition were fitted back to the original EEG of each subject, including all data points (not only GFP peaks), except for periods that were marked as artefacts. Back-fitting means that the spatial correlation between the cluster maps and each individual data point was calculated and the data point was labelled with the cluster map that showed the highest correlation (winner takes all, see e.g. (Britz et al., 2010; van de Ville et al., 2010)). Note that the polarity of the maps was again ignored in this back-fitting procedure. Data points where none of the cluster maps reached a correlation higher than $50 \%$ were labelled as "non-assigned". Once the whole recording was labelled, a temporal smoothing was applied by ignoring segments where a given cluster map was present for less than 4 time points $(32 \mathrm{~ms})$ and the time points were split and assigned to the preceding and following cluster map. Two temporal parameters were then quantified for each recording of each subject: the mean duration a given cluster map (microstate) was present without interruption, and the number of times per second a given microstate appeared, independent of the duration - termed "occurrence" (Michel and Koenig, 2018). In addition, we analysed the syntax of EEG microstates by computing the probabilities from a single $n^{\text {th }}$-previous state to the current one for each subject and transition pair, normalized by the occurrence of each state (Lehmann et al., 2005; Tomescu et al., 2018)).

\subsubsection{EEG source localization}

In order to estimate the sources contributing to each of the microstates, we calculated a distributed linear inverse solution (LAURA) (Grave de Peralta Menendez et al., 2004). The lead field for the inverse solution was calculated for 64 electrode positions and the average brain of the Montreal Neurological Institute, in a grey matter-constrained head model using the LSMAC head model with 5000 distributed solution points (Brunet et al., 2011). A standardization across time was applied for each solution point in order to eliminate activation biases (Supplementary Method). The estimated current densities of each subject were then averaged across all time points that were attributed to a given microstate in each condition.

\subsubsection{Anatomical overlap between fMRI connectivity-derived sub-networks and EEG microstates}

In order to evaluate the anatomical correspondence between the estimated sources of the EEG microstates and the fMRI connectivityderived sub-networks, we estimated the volumes of spatial overlap between the two modalities. The EEG microstate source distributions were first converted to binary masks by selecting only the areas with activity above the 97th percentile to minimize the smearing inherent to the source localization algorithm. These cluster masks were then directly compared to the ROIs resulting from the cluster-based fALFF analysis, and particularly the networks across ROIs that were identified with the connectivity analysis. The volumes of spatial overlap were thereby estimated in terms of their binary intersection.

\subsubsection{Statistical analysis}

The cluster maps derived for each of the three conditions (rest, memory and math) were ordered according to their highest spatial correlation across conditions, and labelled according to the canonical microstate maps described in the literature (Michel and Koenig, 2018). In order to evaluate whether the microstate maps with the same labels were generated by similar networks, we compared the estimated sources of each cluster map between the three conditions. For that, paired t-tests were calculated for each solution point across subjects and Bonferroni-corrected for multiple testing.

To investigate differences in the temporal dynamics (duration, occurrence) of the microstates between conditions we performed repeated measure ANOVAs with the factors "condition" (rest, math, memory) for each microstate class. We applied a threshold of $p<0.0001$ to correct for multiple testing and performed post-hoc comparisons only if this threshold was reached.

The transition probabilities between microstates were statistically compared within and between each condition using paired t-tests.

\section{Results}

\subsection{Instructed thoughts modulate fMRI networks}

Across all subjects and runs, the range of head motion was found to be below $0.5 \mathrm{~mm}$ and $0.09 \mathrm{~mm}$ on average for absolute and relative head displacement, respectively (as reported by FSL's motion estimation step) - therefore well below the voxel size. No significant changes in absolute or relative head displacement were found across the three conditions ( $\mathrm{p}=0.09$ and $\mathrm{p}=0.10$, respectively, 1 -way ANOVA).

To identify changes in brain activity across different brain regions during the two task-initiated, self-generated processing conditions (math and memory), fALFF values were mapped (Zou et al., 2008), serving as a model-free measure of local BOLD activity (Biswal, 2012). Having obtained a fALFF estimate for each brain voxel of each fMRI run and participant, these values were then compared between conditions on the group level. Condition-specific fALFF changes were found for several cortical and subcortical brain regions. The regions with significantly higher brain activity during the memory compared to the math condition (Fig. 2a) included areas in the parietal, the medial temporal, the prefrontal and occipital lobes. We found dominant activity in the lateral part of the parietal lobe: bilaterally the supramarginal gyrus (lrSMG, BA40) and the right angular gyrus (rAG, BA39) of the inferior parietal lobe (IPL). Activity was also found in the medial part of the parietal lobe: the left precuneus (1PCu, BA7), the dorsal part and the ventral part of the posterior cingulate cortex/retrosplenial cortex (dPCC, BA31; vPCC/RSc, 

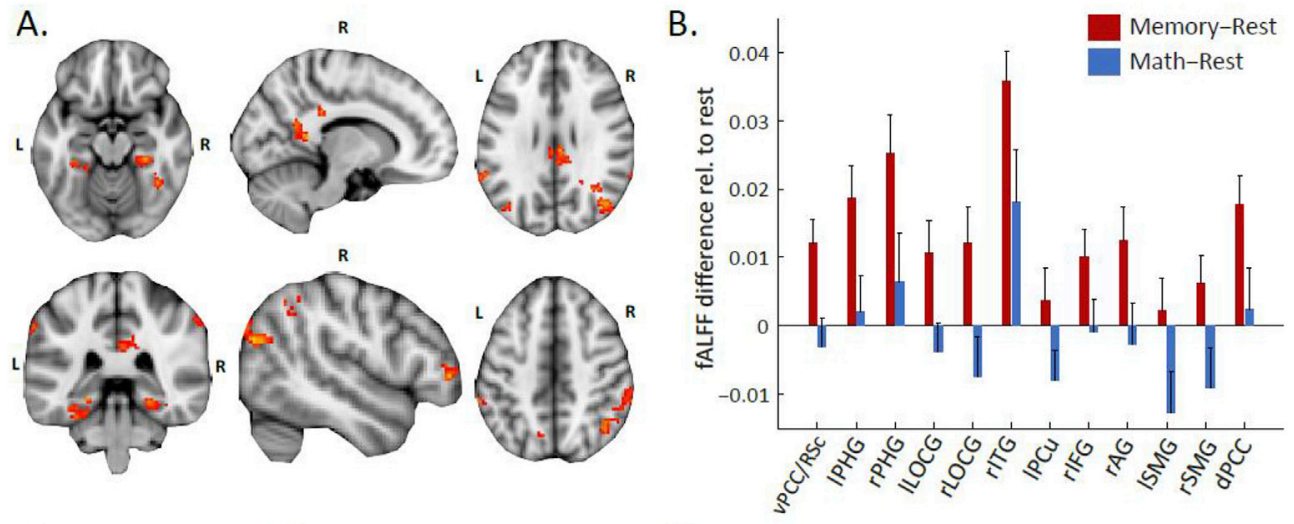

C.

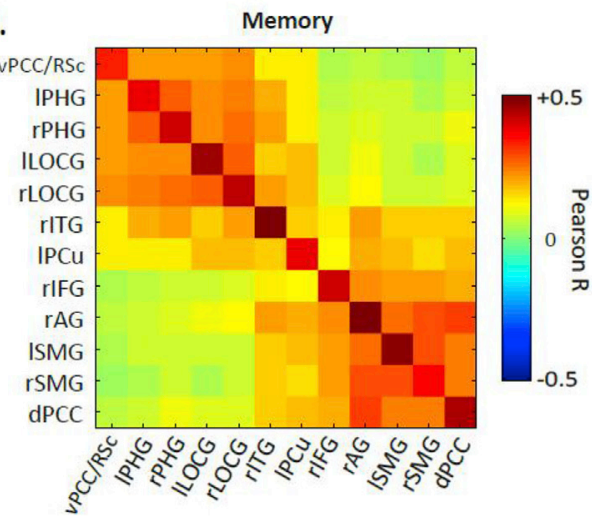

B.

D.

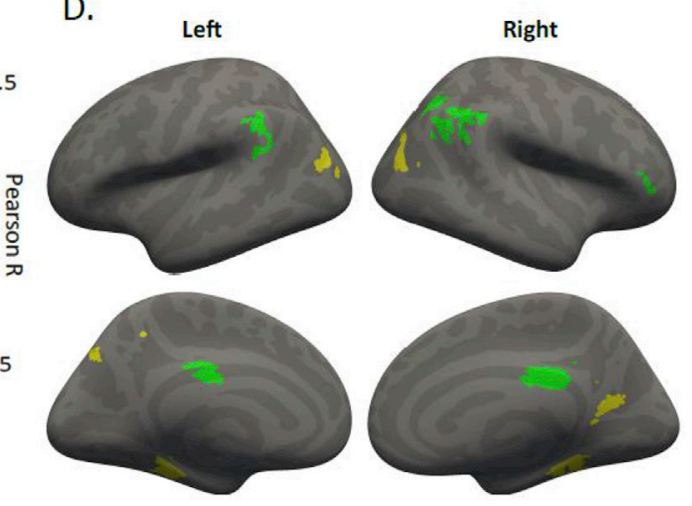

Fig. 2. Memory-specific changes in fMRI activity and fMRI functional connectivity memory sub-networks.

BA23. In the temporal lobe we found bilaterally-increased activity in the parahippocampal gyri (lrPHG, BA28,35,36) and the right inferior temporal gyrus (rITG, BA21). Significant activity was also found in the right inferior frontal gyrus (rIFG, BA45), and bilaterally in the lateral occipital gyri (lrLOCG, BA19). When comparing the fALFF estimates in these brain regions to the non-instructed rest condition (considered as baseline) we found that some of the memory-math differences were due to a stronger increase in BOLD activity during the memory compared to math condition (areas included lrPHG and dPCC), while other changes were due to increased BOLD activity during the memory and decreased BOLD activity during the math conditions. Areas de-activated during math compared to rest included rAG, lrSMG, lrLOCG, IPCu and vPCC/RSc. (Fig. 2b). Conversely, we also identified a set of brain areas significantly more active during the math compared to the memory condition: in the frontal lobe, significantly higher activity was found in the ventral and dorsal anterior cingulate cortex and bilaterally in the dorsolateral prefrontal cortex. Areas in the temporal lobe included bilaterally the superior temporal gyrus and the left middle temporal gyrus. In the parietal cortex, significantly higher activity was found in the right intraparietal sulcus and the pre/post central gyrus as well as the dorsal posterior cingulate cortex. In the occipital cortex the occipital gyrus was activated bilaterally. In contrast to the above observation of areas de-activated during math (compared to the rest condition), none of the areas with significantly higher activity in the math condition were actually de-activated during the memory condition (Supplementary Figs. S2a and S2b). In light of recent evidence indicating that temporal de-trending in fMRI pre-processing can negatively affect fALFF analyses at 7T (Woletz et al., 2018), an additional test was performed whereby the ROI-specific fALFF estimates of Fig.2b and Fig. S2b were repeated when using a more permissive de-trending cut-off (200s, instead of 100s). This produced small-to-negligible changes in the order of $2.4 \%$ on average across ROIs, and without a systematic behaviour (some ROIs showing increases, other decreases). This strongly indicated that the de-trending did not have appreciable effects on the analysis. To determine condition-specific networks, we further analysed, in terms of their functional connectivity, the brain regions that demonstrated significant BOLD activity changes between the memory and math conditions, as identified by the fALFF analysis. These connectivity values were organized in matrices, and the regions were re-ordered according to their correlation profiles. The connectivity analysis revealed two distinct networks within the regions that displayed stronger activity during the memory condition (Fig. 2c and d). Network I. included the vPCC/RSc, lrPHG, lrOCG, while network II. comprised the rIFG, rAG, lrSMG, and dPCC. Two areas (rITG and IPCu) were included in both networks. Compared to the non-instructed rest condition, both networks displayed strong increases in connectivity amidst their respective regions, while remaining uncorrelated to each other and to the areas identified in the math condition.

A. fMRI fALFF analysis. T-test of fALFF for memory $>$ math condition revealed stronger activity in lrSMG, rAG, lrLOCG, lPCu, dPCC, vPCC/ RSc, lrPHG, rITG, rIFG. T-score threshold: T $>2.20$; FDR-corrected for multiple comparisons (5\%). B. Areas of stronger activity in the memory, compared to math condition. Some of the memory-math differences were due to a stronger increase in BOLD activity during the memory than the math condition relative to rest, while other changes were due to increased BOLD activity during the memory and decreased BOLD activity during the math conditions relative to rest. C. Connectivity analysis. Network I. included the vPCC/RSc, lrPHG, lrOCG. Network II. comprised the rIFG, rAG, lrSMG, and dPCC. The rITG and IPCu were found in both subnetworks. D. Sub-networks of the connectivity analysis. Regions were also projected onto a surface template for better visualization.

\subsection{Instructed thoughts modulated the temporal dynamics of specific EEG microstates}

A k-means cluster analysis was first applied to each individual subject and then across subjects within each condition. While the optimal number of clusters varied between subjects, the meta-criterion 
determined 6 clusters as the optimal number in the clustering across subjects for each condition. The topographies of these six maps were strikingly similar between the three conditions and resembled those previously described in the literature (Michel and Koenig, 2018). We ordered the microstate maps according to their highest spatial correlation across conditions (i.e. math, memory, rest) and labelled them from A-F states (Custo et al., 2017; Michel and Koenig, 2018) (Fig. 3a). Statistical comparison of the underlying brain sources that generated these microstates indicated similar networks between the corresponding maps in the three conditions while different microstate maps were characterized by highly significantly different networks (Supplementary Table S1). We then fitted the maps back to the original EEG of each participant (by ignoring polarity) and labelled each time point with the microstate that had highest spatial correlation (Fig. 3b). This procedure allowed us to determine the mean duration of each microstate in the three conditions, and how often the microstates occurred independent of their duration.

ANOVA analyses for each microstate revealed that two states (i.e. microstates $\mathrm{C}$ and D) significantly differed in their duration and occurrence depending on the condition (Fig. 3c) and one state (i.e. microstate B) significantly differed in its occurrence (Fig. 3d). Microstate B significantly increased in occurrence compared to the math as well as to the rest conditions (both $\mathrm{p}<0.0001$ ). Microstate $\mathrm{C}$ significantly decreased in duration and occurrence in the math condition as compared to both, the memory and the rest conditions (both $\mathrm{p}<0.001$ ). Finally, microstate $\mathrm{D}$ significantly increased in occurrence and duration compared to the memory $(\mathrm{p}<0.001)$ and the rest $(\mathrm{p}<0.0001)$ conditions.

A. The six microstates identified by k-means cluster analysis across subjects in the three conditions (rest, memory and math). B. A representative period of 10 -sec EEG with eyes closed after picture presentation is shown (traces of 64 electrodes and Global Field Power (GFP) trace). Back-fitting the 6 microstate maps derived from the cluster analysis shows the chunking of the EEG into segments of various durations covered by one of the microstate maps (indicated by different colors). C. Mean and standard error of the duration of each of the microstates in each condition. Post-hoc tests were performed when the ANOVA revealed significant condition differences at $\mathrm{p}<0.0001$. This was the case for microstate map C and map D: Microstate C was significantly shorter and microstate D significantly longer in the math condition compared to memory and rest. D. Occurrence of the six microstates in the three conditions (number of microstates per second). Significant ANOVAs were found for microstates B, C, and D. As for the duration, microstate $\mathrm{C}$ occurred less and microstate $\mathrm{D}$ more often in the math condition compared to rest and memory. Concerning microstate B, an increased occurrence was found for memory compared to math and rest.

\subsection{Sources of EEG microstates}

To estimate networks underlying each microstate we inverted the original data of each subject into source space using a distributed linear inverse solution (Grave de Peralta Menendez et al., 2004), as described in the Methods. We then averaged the estimated activity across all time points that were labelled with the same microstate maps for each subject and each condition. The brain regions underlying the six microstate maps confirmed and extended previous efforts (Custo et al., 2017) in source localization of microstates (Fig. 4 and Supplementary Figs. S3-S5). Specifically, microstate A showed left-lateralized activity in the superior temporal gyrus (STG), the medial prefrontal cortex (MPFC) and the OCG. Microstate B showed main activity in OCG and in the medial part of the parietal cortex in the PCu/RSc. The sources of microstate $\mathrm{C}$ were located bilaterally in the lateral part of the parietal cortex including both the SMG and AG. The sources of microstate D showed main activity bilaterally in the IFG, dACC, and superior parietal lobule (SPL)/intraparietal sulcus (IPS). Strongest activity for microstate $\mathrm{E}$ was found in the right MPFC. Finally, microstate F showed bilateral activity in the MPFC.

The EEG of each participant and condition were subjected to a distributed linear inverse solution and standardized across time. The source maps of all time points that were labelled with the same microstate map were then averaged within participants. The mean sources across subjects in the memory condition are illustrated here (for individual source maps per condition see Supplementary Figs. S3-S5). Areas
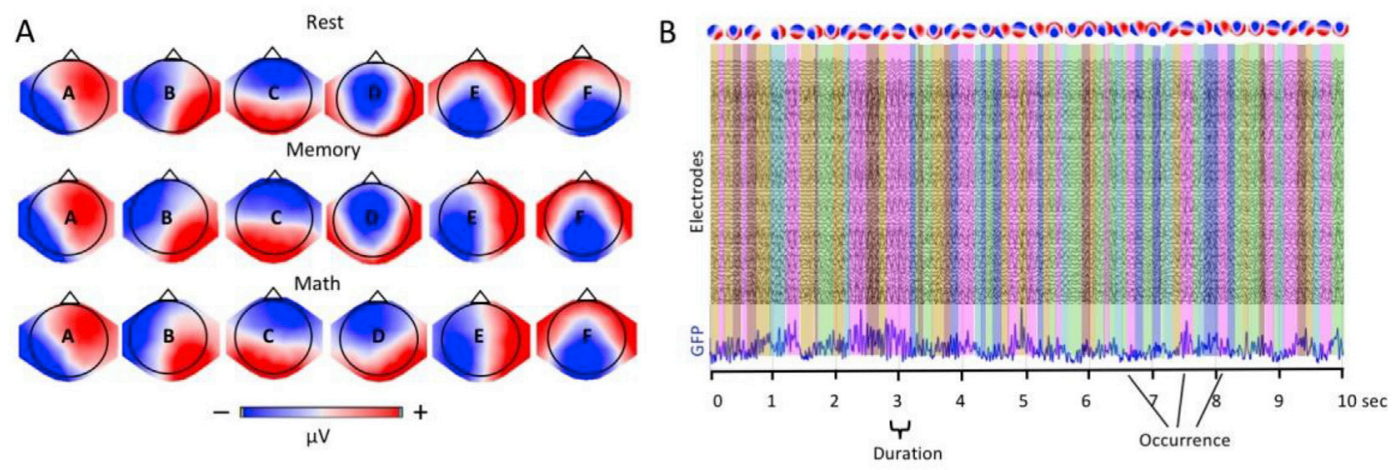

C

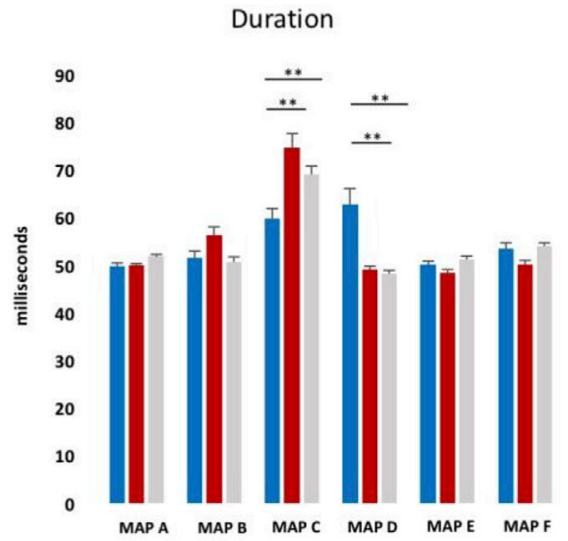

D

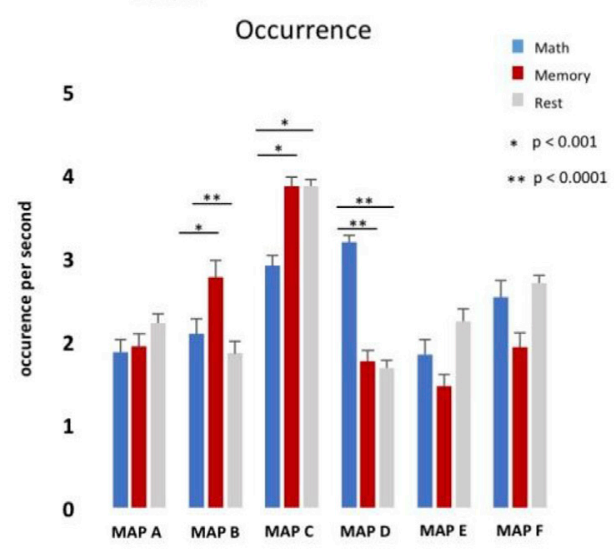

Fig. 3. Temporal dynamics of EEG microstates. 
A
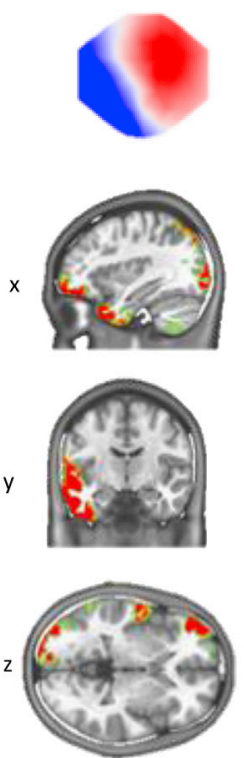

$X=-38, y=-12, z=-1$
B
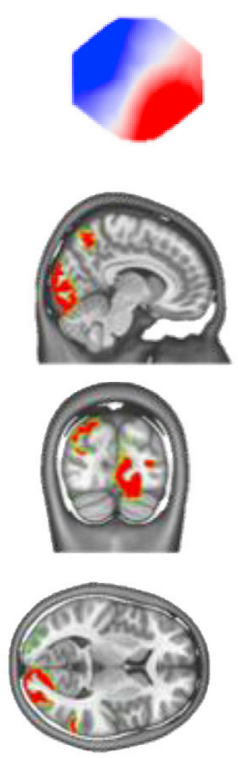

$x=7, y=-80, z=6$
C
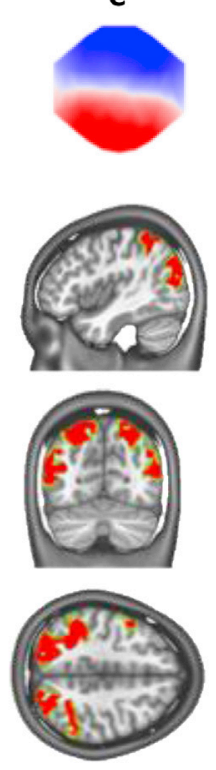

$x=-43, y=-69, z=46$
D
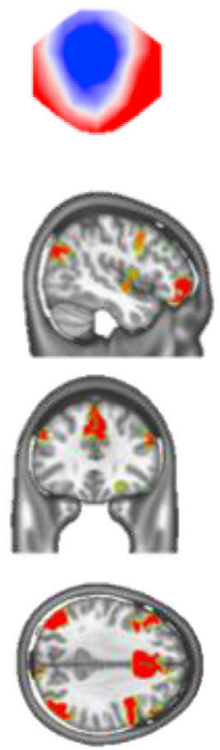

$x=-40, y=32, z=33$
E
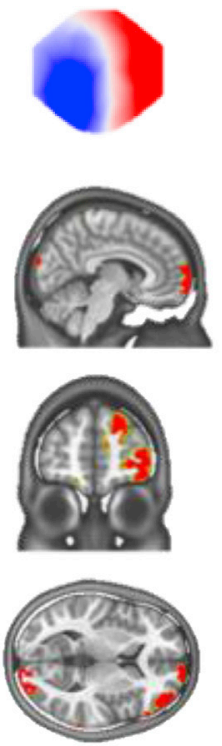

$x=3, y=52, z=5$
$F$
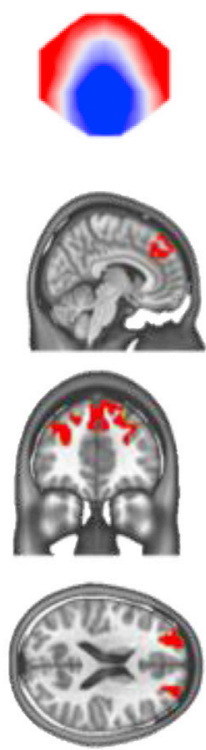

$x=5, y=42, z=21$

Fig. 4. Source localization of EEG microstates.

with activity above 95 percentiles are shown. Notice the strong activity of the superior parietal lobe for microstate $\mathrm{C}$ (strong presence in memory and rest) and the frontoparietal activity for microstate $\mathrm{D}$ (strong presence in math).

\subsection{Transition between microstates}

To determine whether the brain alternates between the two dominating microstates in the memory condition (microstates $\mathrm{B}$ and $\mathrm{C}$ ), we calculated the transition probabilities (normalized by the occurrence) between microstate $\mathrm{B}$ and $\mathrm{C}$ and compared them to all other possible transitions. We found that specifically and only in the memory condition, the transition from microstate $\mathrm{C}$ to microstate $\mathrm{B}$ was significantly more frequent than the transition from any other state and that microstate B transitioned significantly more often to microstate $\mathrm{C}$ than to any other state (Fig. 5). Likewise, microstate $C$ was significantly more often followed by microstate B than microstates A, D or E, but not F. The same analysis was applied to the transition probabilities in the math and rest conditions (see Supplementary Figs. S6 and S7). In the rest condition, no specific microstate was preceding or following microstate B. In the math condition, microstate $\mathrm{C}$ was significantly less frequently preceding or following microstate $\mathrm{C}$ compared to all other microstates.

We calculated the transition probabilities from each microstate to any other using Markov chains. The observed probabilities were divided by the expected probabilites to account for the variability in occurrence of the states. In the memory condition, we found that microstate $B$ was significantly more often preceded (A) and followed (B) by microstate C than any of the other microstates. This transition behaviour was not found during rest (Supplementray Fig. S6) or when participants performed mental arithmetic operations (Supplementray Fig. S7).

We also compared all transition probabilities between the conditions using paired t-tests (Fig. 6). This analysis confirmed the increased transition between microstate $\mathrm{B}$ and $\mathrm{C}$ in the memory condition. In the math condition, an increased transition to microstate $\mathrm{D}$ was found, while in the rest condition the transition between microstate $\mathrm{E}$ and $\mathrm{C}$ was increased compared to the math condition.

Paired t-tests of all transition probabilities between the different conditions revealed increased transitions between microstate $\mathrm{B}$ and $\mathrm{C}$ in
A

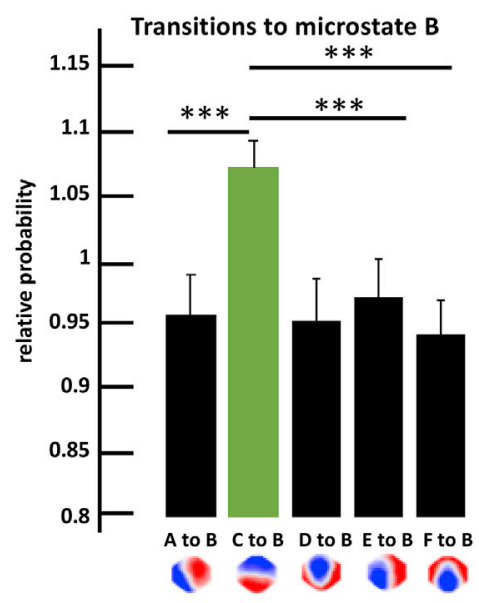

B

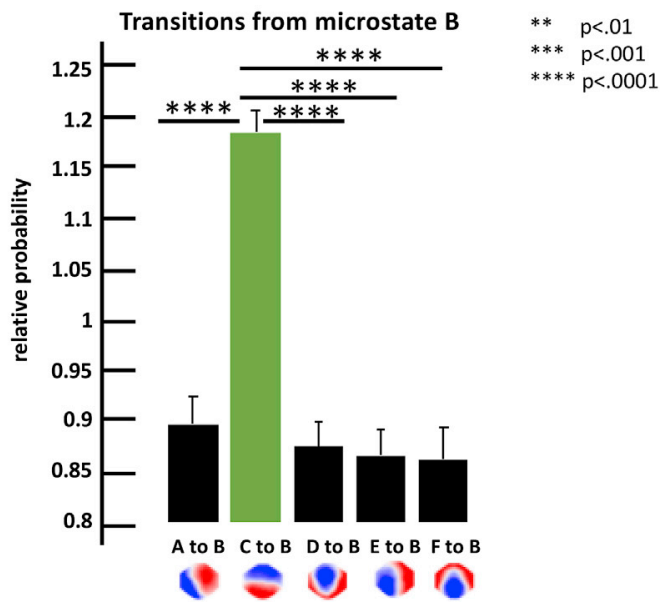

Fig. 5. Markov chain transition probabilities in the memory condition. 


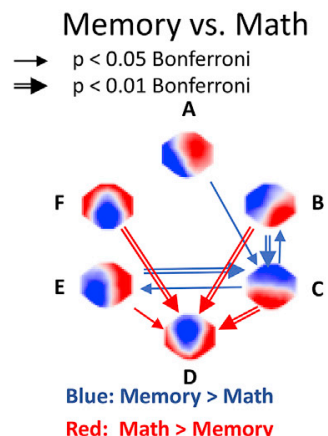

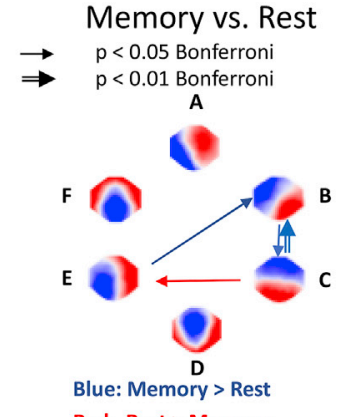

Red: Rest $>$ Memory

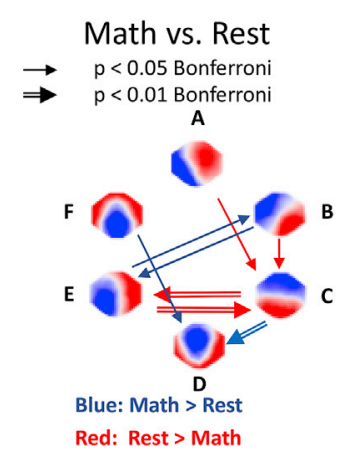

Fig. 6. Comparison of the transition probabilities between the three conditions.

the memory condition (compared to Math and Rest) and increased transitions to microstate D in the Math condition compared to Memory. During Rest compared to Math, increased transitions between microstate $\mathrm{C}$ and $\mathrm{E}$ were found.

\subsection{Anatomical overlap between fMRI-derived sub-networks and EEG microstates}

We found an anatomical overlap between the fMRI-derived subnetwork I and EEG microstate B in 154 voxels corresponding to a volume of $1232 \mathrm{~mm}^{3}$. The area of overlap was found in the lateral parts of the lateral occiptal gyrus (Fig. 7A). For EEG microstate C, we found an overlap with the fMRI-derived sub-network II in 73 voxels corresponding to a volume of $584 \mathrm{~mm}^{3}$. This overlap was found bilaterally in the inferior parietal lobule (Fig. 7B). Importantly, even when applying a more restrictive cluster-forming threshold of $\mathrm{T}>3.0$ in the fALFF analysis, the surviving area in subnetwork $I$ is the lateral occipital cortex, and the surviving area in subnetwork II is inferior parietal lobule, i.e. the two areas that overlay with the maximal sources of EEG microstates B and C, respectively (see Supplementary Fig. S8).

A. Visualization of the anatomical overlap between the fMRI connectivity-derived sub-network I and EEG microstate B showed a core overlap in the lateral occipital gyrus. B. Visualization of the anatomical overlap between fMRI sub-network II and EEG microstate C showed a core overlap in the inferior parietal lobule.

\section{Discussion}

By using fMRI and EEG in a paradigm where participants were instructed to focus their thoughts on specific tasks, we here provide direct evidence of capturing the temporal occurrence of specific large-scale brain networks that are involved in self-related and self-unrelated thoughts. In line with previous task-related studies (Andrews-Hanna, 2012; Andrews-Hanna et al., 2014; Axelrod et al., 2017), the fMRI data revealed that thinking about self-experienced past events boosted a brain network known to be related to autobiographic episodic memory, while performing mental arithmetic enhanced a network known to be involved in attention and cognitive control. Intriguingly, the EEG analysis showed that the different conditions produced modulations in the occurrence and duration of brief, sub-second episodes of stable configuration of the brain electric field (the EEG microstates). Source localization of these episodes revealed networks that spatially coincided to those observed with fMRI.

Crucially, in this study, we sought to build upon previous fMRI work that applied experimental designs with faster alternating conditions (each lasting for less than 30s (Axelrod et al., 2017), to explore a design where all trials of each condition are grouped in an individual run lasting for several minutes. This allowed not only the study of changes in BOLD activity for each brain region (through fALFF analysis), but also the study of the interactions between those regions across time (through functional connectivity analysis) throughout the same condition - thereby shedding new light on the question of whether different functional regions that
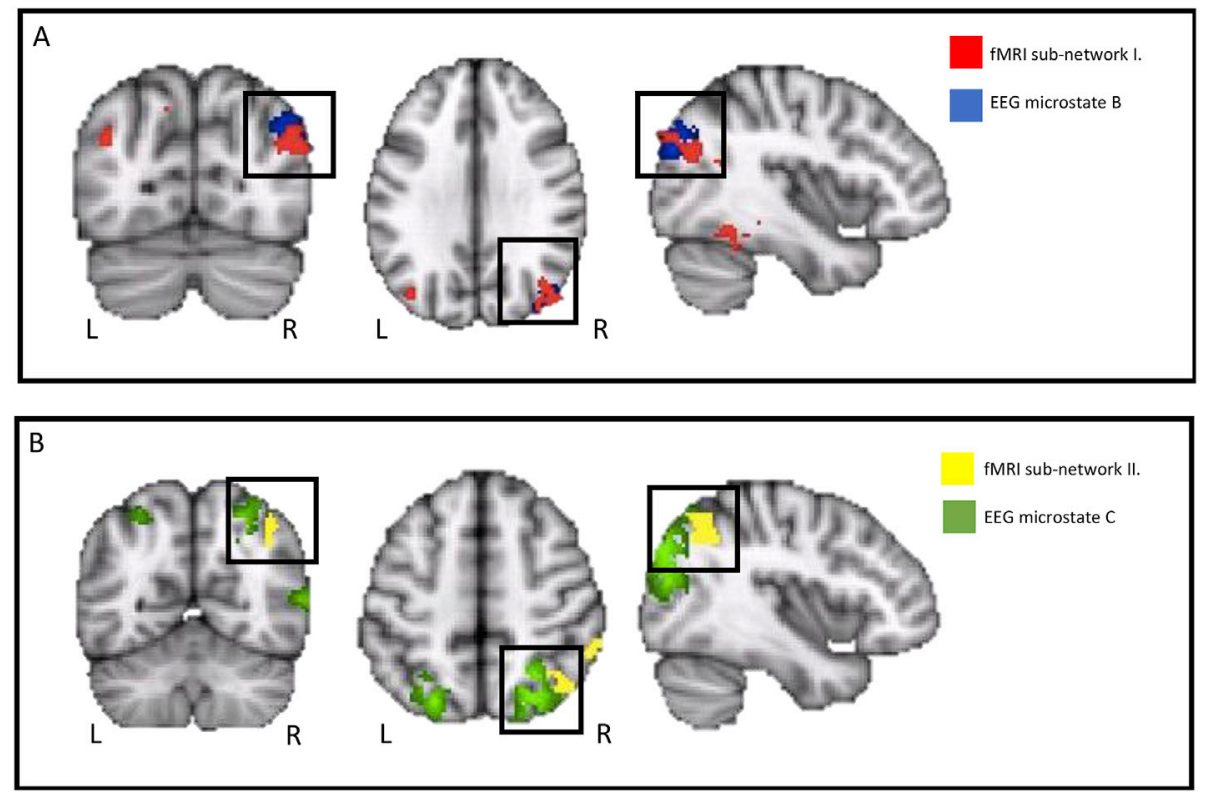

Fig. 7. Anatomical overlap between fMRI-derived sub-networks and EEG microstates. 
become active at the same time effectively share similar BOLD activity.

The fALFF analysis confirmed and extended previous studies by revealing condition-specific BOLD changes in several cortical and subcortical brain regions. The regions with significantly higher brain activity during the memory, compared to the math condition, included the core brain areas of the episodic memory retrieval network (Schacter et al., 2012; Spreng and Grady, 2010; Svoboda et al., 2006) in the parietal, the medial temporal, the prefrontal and occipital lobes. Conversely, we also identified a set of brain areas more active during the math, compared to the memory condition, that have been previously associated to mental arithmetic tasks and frontoparietal control network (FPCN) in general (Fehr et al., 2008).

The fMRI connectivity results revealed that the regions of increased fALFF were essentially organized in two subsystems with distinct functional and anatomical characteristics - highly correlated within themselves, but poorly correlated between each other. Network I. was strikingly similar to a previously described subsystem which becomes involved during constructions of mental scenes (hereafter termed "scenereconstruction subsystem"), while network II. closely matched a previously defined subsystem which becomes active when participants engage in self-relevant cognitive processes and reflect on their current mental states (hereafter termed "self-experience subsystem") (Andrews-Hanna et al., 2010a, 2014; Axelrod et al., 2017).

Beyond the slower timescale of fMRI measures, the EEG microstate analysis revealed new insights into the temporal dynamics of these networks in the sub-second range. We found that specific EEG microstates, representing brief periods of coherent network activity, were selectively increased in duration and occurrence by the instructed thoughts. The brain regions generating these microstates partly overlapped with the specific fMRI networks. The network underlying microstate $\mathrm{C}$, which occurred more often and lasted longer in the memory compared to the math condition, overlapped with the lateral parietal areas of the "selfexperience" memory retrieval subnetwork that we identified in the fMRI analyses. On the other hand, the brain areas underlying microstate B, which selectively increased in occurrence during the memory condition, overlapped with the lateral occipital areas of the "scene-reconstruction" subnetwork that we detected in the fMRI analyses. Finally, brain areas underlying microstate $\mathrm{D}$, which selectively increased in duration and occurrence in the math condition, included areas generally attributed to the frontoparietal control network (FPCN) (Ptak et al., 2017). Moreover, during memory retrieval, we found increased transitions between the microstates that corresponded to the "self-experience" and "scene-reconstruction" subnetworks in the fMRI data. These results provide direct evidence that the RSNs captured by fMRI are tightly linked to, and possibly originated by, a prolongation and repeated occurrence of states of synchronized activity of specific large-scale neuronal networks, in the sub-second timescale.

To capture the occurrence of any conscious experience, and to directly investigate the cognitive processes operating during mindwandering, it is crucial to better control the spontaneous thoughts of participants (Koch, 2018; Kucyi et al., 2016). In line with other recent studies (Andrews-Hanna et al., 2010b; Axelrod et al., 2017; Delamillieure et al., 2010), we here initiated periods of spontaneous mentation with brief presentations of external stimuli and instructed participants to close their eyes and internally direct their thoughts to either self-related photographs or self-unrelated arithmetic operations. The main regions that showed increased BOLD activity in the memory condition comprise the IPL and MTL structures. Numerous studies have previously identified these regions as the core of the episodic memory retrieval network (Schacter et al., 2012; Spreng and Grady, 2010; Svoboda et al., 2006). Traditionally, since the discovery of densely amnesic patients, the MTL structures have been regarded as essential for long-term memory formation, allowing us to remember past experiences and to retrieve acquired knowledge (Moscovitch et al., 2016; Steinvorth et al., 2005). In contrast to the MTL structures, extensive evidence from patient lesion and brain stimulation studies suggests that the IPL plays a key role in integrating vivid details of personally experienced events into conscious representations during memory retrieval (Berryhill et al., 2007; Simons et al., 2010). The subjective experience of perceiving a scene, recognizing a face, hearing a sound or reflecting on the experience itself presents a complex interplay between memory, attention and consciousness (Corbetta and Shulman, 2011; Tononi et al., 2016). Tulving (1985) defined the underlying ability to re-experience the subjective sense of self in the past and to mentally project oneself into the future, i.e. the autonoetic consciousness, as the crucial aspect of episodic memory retrieval. Evidence across lesion studies, stimulation and recording studies consistently point to the posterior regions, including temporal, parietal and occipital areas ("posterior hot zone") as playing a direct role in specifying the contents of consciousness (Koch, 2018). Our current findings are also in line with a recent study (Brechet et al., 2018) where we confirmed the contribution of the IPL, especially the AG, to the subjective, first-person perspective re-experience of self-relevant, vivid past episodes.

Brain activity constantly fluctuates in and out of different mental states that are stable for fractions of seconds. Only one epoch or state of conscious content can be considered at a time (Seth and Baars, 2005). It is assumed that the EEG microstates capture these states that last for around $100 \mathrm{~ms}$ only (Baars, 2002a; Changeux and Michel, 2004). Using high-density whole-brain EEG, we indeed observed modulations of duration, occurrence, and transitions between particular microstates by the instructed thoughts, microstate $\mathrm{B}$ and $\mathrm{C}$ being increased in the memory condition and microstate $\mathrm{D}$ being increased in the math condition. The topographies of these three microstates strongly resemble three of the four canonical microstates previously described in the literature (for reviews see (Khanna et al., 2015; Michel and Koenig, 2018)). Studies on large cohorts showed that microstate $\mathrm{C}$ is generally the most dominant state during eyes-closed rest (Koenig et al., 2002; Tomescu et al., 2018). Recently, Seitzman et al. (2017) showed that microstate C decreases in duration and occurrence during a mental arithmetic task, similar to our findings. Decrease of microstate C duration has also been described when subjects are engaged in object or verbal visualization tasks compared to rest (Milz et al., 2016b). Based on these and other studies, it is assumed that microstate C reflects activity in the DMN (Custo et al., 2017; Milz et al., 2016b; Panda et al., 2016; Seitzman et al., 2017); for a discussion, see (Michel and Koenig, 2018). Indeed, our analysis of the sources underlying this microstate confirms such interpretation. The sources of microstate $\mathrm{C}$ were located bilaterally in the lateral part of the parietal lobe and MTG, areas that we attributed in the fMRI results to the "self-experience subsystem". The observation that microstate C is not significantly boosted by the memory condition compared to rest is not surprising. It confirms the assumption that self-relevant memory retrieval also predominates during spontaneous mind wandering.

Moreover, our investigation revealed that microstate B increased in occurrence during the memory as compared to both rest and math conditions. This state has been previously attributed to the visual network (Britz et al., 2010; Custo et al., 2014, 2017). For example, Milz and colleagues (Milz et al., 2016b) showed that microstate B increased when participants were asked to visualize previously presented images. Source localization in our study also indicated strong activation of the visual cortex together with medial parietal areas. Intriguingly, lesions to the medial parietal cortex cause memory recognition and visuospatial impairments, but no impairments related to self-consciousness (Davidson et al., 2008). We thus interpret the increased occurrence of microstate $B$ as related to the "scene-reconstruction subsystem" found in the fMRI connectivity analysis. An intriguing assumption is therefore that the microstate analysis allows us to disentangle the sub-parts of thoughts related to the conscious experience of an episodic autobiographic memory, i.e. visualization of the scene and visualization of the self in the scene. Indeed, the transition probability analysis revealed a more frequent switching between microstate $\mathrm{B}$ and $\mathrm{C}$ than between any other state.

Additionally, our results show that while both microstates B and C are less frequently appearing when participants are engaged in math 
calculations, microstate D strongly increases in duration and occurrence during this condition. Microstate D has previously been attributed to the attention/cognitive control network including frontoparietal areas (Britz et al., 2010; Custo et al., 2017) and our source localization of microstate D confirmed the attribution of this state to the frontoparietal control network (FPCN) (Ptak et al., 2017). Furthermore, the observation of increase of microstate $\mathrm{D}$ and decrease of microstate $\mathrm{C}$ fits very well to the observation that the FPCN and the DMN are inversely activated when participants are engaged in external-directed and self-directed cognition (Dixon et al., 2018). Therefore, we show that the large-scale network anti-correlation found in the fMRI data is associated with sub-second modulation of the presence of microstates sub-serving these functions.

EEG microstate studies have repeatedly revealed changes of the temporal dynamics of microstates in mental disease, particularly schizophrenia (for reviews see (Khanna et al., 2014; Michel and Koenig, 2018). The most robust finding, confirmed in a recent meta-analysis (Rieger, 2016), is an increase in duration and occurrence of microstate $\mathrm{C}$ and a decrease of microstate D in patients with schizophrenia (Koenig et al., 1999; Lehmann et al., 2005) or at risk to develop schizophrenia (Andreou et al., 2014; Tomescu et al., 2014), a disequilibrium that is normalized when patients are treated with antipsychotic medication (Kikuchi et al., 2007) and with rTMS (Sverak et al., 2017). These findings correspond well to the interpretation that microstate $\mathrm{C}$ reflects introspective, self-focused thoughts, while microstate D reflects attention and cognitive control. An increase of microstate $C$ and decrease of microstate $\mathrm{D}$ in schizophrenia might index the progressive detachment of mental states from environmental input. While a healthy person constantly and effortlessly balances periods of rest with periods of focused attention when interacting with their surroundings, patients with schizophrenia or other mental disorders may persist on thinking about a particular unpleasant event that involves themselves and lose control over the natural flow of the wandering mind. Understanding the functional significance of microstates with studies as the one presented here might thus not only be relevant for monitoring the vulnerability of patients at risk for mental disease and the effects of treatment, but also for better understanding the thoughts that these patients are caught in.

The microstate model assumes that an EEG time series can be described as a sequence of a finite number of states and that at each time point, only one state is active. This is implemented in the winner-takes-all approach by labeling each time point with only one cluster map. In practice, there is a certain degree of overlap and other states also explain a certain amount of variance at a given time point. The microstate model is admittedly a simplification, but it allows to temporally resolve restingstate data into elements that have the potential to represent basic steps of information processing. These elements can be achieved by modeling the data using a sequence of non-overlapping, quasi-stable states. This global, extremely simple, and data-driven method can be used to extract unique features such as the duration and occurrence of these states. In addition, the microstate methodology is embedded in the general functional theory that assumes that only one functional state occurs at any given moment in time (Baars, 2002b; Meehan and Bressler, 2012; Seth and Baars, 2005).

\section{Acknowledgments}

This work was supported by the Swiss National Science Foundation (grant No. 320030_159705) to CMM and the National Centre of Competence in Research (NCCR) "SYNAPSY-The Synaptic Basis of Mental Diseases" (NCCR Synapsy Grant \# “51NF40-185897) to CMM.

\section{Appendix A. Supplementary data}

Supplementary data to this article can be found online at https://doi. org/10.1016/j.neuroimage.2019.03.029.

\section{References}

Andersson, J.L., Skare, S., Ashburner, J., 2003. How to correct susceptibility distortions in spin-echo echo-planar images: application to diffusion tensor imaging. Neuroimage 20, 870-888.

Andreou, C., Faber, P.L., Leicht, G., Schoettle, D., Polomac, N., Hanganu-Opatz, I.L., Lehmann, D., Mulert, C., 2014. Resting-state connectivity in the prodromal phase of schizophrenia: insights from EEG microstates. Schizophr. Res. 152, 513-520.

Andrews-Hanna, J.R., 2012. The brain's default network and its adaptive role in internal mentation. Neuroscientist 18, 251-270.

Andrews-Hanna, J.R., Reidler, J.S., Huang, C., Buckner, R.L., 2010a. Evidence for the default network's role in spontaneous cognition. J. Neurophysiol. 104, 322-335.

Andrews-Hanna, J.R., Reidler, J.S., Sepulcre, J., Poulin, R., Buckner, R.L., 2010b. Functional-anatomic fractionation of the brain's default network. Neuron 65, $550-562$.

Andrews-Hanna, J.R., Saxe, R., Yarkoni, T., 2014. Contributions of episodic retrieval and mentalizing to autobiographical thought: evidence from functional neuroimaging, resting-state connectivity, and fMRI meta-analyses. Neuroimage 91, 324-335.

Axelrod, V., Rees, G., Bar, M., 2017. The default network and the combination of cognitive processes that mediate self-generated thought. Nat Hum Behav 1, 896-910.

Baars, B.J., 2002a. Atoms of thought. Science and Consciousness Review December 1-2.

Baars, B.J., 2002b. The conscious access hypothesis: origins and recent evidence. Trends Cognit. Sci. 6, 47-52.

Berryhill, M.E., Phuong, L., Picasso, L., Cabeza, R., Olson, I.R., 2007. Parietal lobe and episodic memory: bilateral damage causes impaired free recall of autobiographical memory. J. Neurosci. 27, 14415-14423.

Biswal, B., Yetkin, F.Z., Haughton, V.M., Hyde, J.S., 1995. Functional connectivity in the motor cortex of resting human brain using echo-planar MRI. Magn. Reson. Med. 34, 537-541.

Biswal, B.B., 2012. Resting state fMRI: a personal history. Neuroimage 62, 938-944.

Brechet, L., Grivaz, P., Gauthier, B., Blanke, O., 2018. Common recruitment of angular gyrus in episodic autobiographical memory and bodily self-consciousness. Front. Behav. Neurosci. 12, 270.

Bressler, S.L., Menon, V., 2010. Large-scale brain networks in cognition: emerging methods and principles. Trends Cognit. Sci. 14, 277-290.

Britz, J., Van De Ville, D., Michel, C.M., 2010. BOLD correlates of EEG topography reveal rapid resting-state network dynamics. Neuroimage 52, 1162-1170.

Brunet, D., Murray, M.M., Michel, C.M., 2011. Spatiotemporal analysis of multichannel EEG: CARTOOL. Comput. Intell. Neurosci. 2011, 813870.

Cabral, J., Vidaurre, D., Marques, P., Magalhaes, R., Silva Moreira, P., Miguel Soares, J., Deco, G., Sousa, N., Kringelbach, M.L., 2017. Cognitive performance in healthy older adults relates to spontaneous switching between states of functional connectivity during rest. Sci. Rep. 7, 5135.

Changeux, J.-P., Michel, C.M., 2004. Mechanism of neural integration at the brain-scale level. In: Grillner, S., Graybiel, A.M. (Eds.), Microcircuits. MIT Press, Cambridge, pp. 347-370.

Charrad, M., Ghazzali, N., Boiteau, V., Niknafs, A., 2014. NbClust: an R package for determining the relevant number of clusters in a data set. J. Stat. Softw. 61.

Christoff, K., Irving, Z.C., Fox, K.C., Spreng, R.N., Andrews-Hanna, J.R., 2016. Mind-wandering as spontaneous thought: a dynamic framework. Nat. Rev. Neurosci. 17, 718-731.

Chumbley, J., Worsley, K., Flandin, G., Friston, K., 2010. Topological FDR for neuroimaging. Neuroimage 49, 3057-3064.

Cole, M.W., Bassett, D.S., Power, J.D., Braver, T.S., Petersen, S.E., 2014. Intrinsic and task-evoked network architectures of the human brain. Neuron 83, 238-251.

Cole, M.W., Reynolds, J.R., Power, J.D., Repovs, G., Anticevic, A., Braver, T.S., 2013. Multi-task connectivity reveals flexible hubs for adaptive task control. Nat. Neurosci. $16,1348-1355$.

Corbetta, M., Shulman, G.L., 2002. Control of goal-directed and stimulus-driven attention in the brain. Nat. Rev. Neurosci. 3, 201-215.

Corbetta, M., Shulman, G.L., 2011. Spatial neglect and attention networks. Annu. Rev, Neurosci. 34, 569-599.

Custo, A., Van De Ville, D., Wells, W.M., Tomescu, M.I., Brunet, D., Michel, C.M., 2017. Electroencephalographic resting-state networks: source localization of microstates. Brain Connect. 7, 671-682.

Custo, A., Vulliemoz, S., Grouiller, F., Van De Ville, D., Michel, C.M., 2014. EEG source imaging of brain states using spatiotemporal regression. Neuroimage 96, 106-116.

Davidson, P.S., Anaki, D., Ciaramelli, E., Cohn, M., Kim, A.S., Murphy, K.J., Troyer, A.K., Moscovitch, M., Levine, B., 2008. Does lateral parietal cortex support episodic memory? Evidence from focal lesion patients. Neuropsychologia 46, 1743-1755.

Davis, S.W., Stanley, M.L., Moscovitch, M., Cabeza, R., 2017. Resting-state networks do not determine cognitive function networks: a commentary on Campbell and Schacter (2016). Lang Cogn Neurosci 32, 669-673.

de Pasquale, F., Corbetta, M., Betti, V., Della Penna, S., 2018. Cortical cores in network dynamics. Neuroimage 180, 370-382.

Deco, G., Jirsa, V.K., McIntosh, A.R., 2011. Emerging concepts for the dynamical organization of resting-state activity in the brain. Nat. Rev. Neurosci. 12, 43-56.

Delamillieure, P., Doucet, G., Mazoyer, B., Turbelin, M.R., Delcroix, N., Mellet, E., Zago, L., Crivello, F., Petit, L., Tzourio-Mazoyer, N., Joliot, M., 2010. The resting state questionnaire: an introspective questionnaire for evaluation of inner experience during the conscious resting state. Brain Res. Bull. 81, 565-573.

Dixon, M.L., De La Vega, A., Mills, C., Andrews-Hanna, J., Spreng, R.N., Cole, M.W., Christoff, K., 2018. Heterogeneity within the frontoparietal control network and its relationship to the default and dorsal attention networks. Proc. Natl. Acad. Sci. U. S. A. 115, E1598-E1607. 
Fehr, T., Code, C., Herrmann, M., 2008. Auditory task presentation reveals predominantly right hemispheric fMRI activation patterns during mental calculation. Neurosci. Lett. 431, 39-44.

Foster, B.L., Dastjerdi, M., Parvizi, J., 2012. Neural populations in human posteromedia cortex display opposing responses during memory and numerical processing. Proc. Natl. Acad. Sci. U. S. A. 109, 15514-15519.

Foster, B.L., Rangarajan, V., Shirer, W.R., Parvizi, J., 2015. Intrinsic and task-dependent coupling of neuronal population activity in human parietal cortex. Neuron 86, 578-590.

Fox, K.C., Spreng, R.N., Ellamil, M., Andrews-Hanna, J.R., Christoff, K., 2015. The wandering brain: meta-analysis of functional neuroimaging studies of mindwandering and related spontaneous thought processes. Neuroimage 111, 611-621.

Fox, K.C.R., Andrews-Hanna, J.R., Mills, C., Dixon, M.L., Markovic, J., Thompson, E., Christoff, K., May 12, 2018. Affective neuroscience of self-generated thought. Ann. N. Y. Acad. Sci. https://doi.org/10.1111/nyas.13740 (EPub ahead of print).

Grave de Peralta Menendez, R., Murray, M.M., Michel, C.M., Martuzzi, R., Gonzalez Andino, S.L., 2004. Electrical neuroimaging based on biophysical constraints. Neuroimage 21, 527-539.

Greve, D.N., Fischl, B., 2009. Accurate and robust brain image alignment using boundarybased registration. Neuroimage 48, 63-72.

Hutchinson, M., Raff, U., 2014. Is the fastest MRI a hologram? J. Neuroimaging 24, 537-542.

Jorge, J., Figueiredo, P., Gruetter, R., van der Zwaag, W., 2018. Mapping and characterization of positive and negative BOLD responses to visual stimulation in multiple brain regions at 7T. Hum. Brain Mapp. 39, 2426-2441.

Jenkinson, M., Bannister, P., Brady, M., Smith, S., 2012. Improved optimization for the robust and accurate linear registration and motion correction of brain images improved optimization for the robust and accurate linear registration and motion correction of brain images. Neuroimage $17,825-841$.

Karahanoglu, F.I., Van De Ville, D., 2015. Transient brain activity disentangles fMRI resting-state dynamics in terms of spatially and temporally overlapping networks. Nat. Commun. 6, 7751.

Khanna, A., Pascual-Leone, A., Farzan, F., 2014. Reliability of resting-state microstate features in electroencephalography. PLoS One 9, e114163.

Khanna, A., Pascual-Leone, A., Michel, C.M., Farzan, F., 2015. Microstates in resting-state EEG: current status and future directions. Neurosci. Biobehav. Rev. 49, 105-113.

Kikuchi, M., Koenig, T., Wada, Y., Higashima, M., Koshino, Y., Strik, W., Dierks, T., 2007 Native EEG and treatment effects in neuroleptic-naive schizophrenic patients: time and frequency domain approaches. Schizophr. Res. 97, 163-172.

Koch, C., 2018. What is consciousness? Nature 557, S8-S12.

Koenig, T., Brandeis, D., 2016. Inappropriate assumptions about EEG state changes and their impact on the quantification of EEG state dynamics. Neuroimage 125, 1104-1106.

Koenig, T., Lehmann, D., Merlo, M.C., Kochi, K., Hell, D., Koukkou, M., 1999. A deviant EEG brain microstate in acute, neuroleptic-naive schizophrenics at rest. Eur. Arch. Psychiatry Clin. Neurosci. 249, 205-211.

Koenig, T., Prichep, L., Lehmann, D., Sosa, P.V., Braeker, E., Kleinlogel, H., Isenhart, R., John, E.R., 2002. Millisecond by millisecond, year by year: normative EEG microstates and developmental stages. Neuroimage 16, 41-48.

Krzanowski, W., Lai, Y., 1988. A criterion for determining the number of groups in a data set using sum of squares clustering. Biometrics 44, 23-34.

Kucyi, A., 2018. Just a thought: how mind-wandering is represented in dynamic brain connectivity. Neuroimage 180, 505-514.

Kucyi, A., Esterman, M., Riley, C.S., Valera, E.M., 2016. Spontaneous default network activity reflects behavioral variability independent of mind-wandering. Proc. Natl, Acad. Sci. U. S. A. 113, 13899-13904.

Lehmann, D., 1990. Brain electric microstates and cognition: the atoms of thought. In: John, E.R. (Ed.), Machinery of the Mind. Birkhäuser, Boston, pp. 209-244.

Lehmann, D., Faber, P.L., Galderisi, S., Herrmann, W.M., Kinoshita, T., Koukkou, M., Mucci, A., Pascual-Marqui, R.D., Saito, N., Wackermann, J., Winterer, G., Koenig, T., 2005. EEG microstate duration and syntax in acute, medication-naive, first-episode schizophrenia: a multi-center study. Psychiatr. Res. 138, 141-156.

Lehmann, D., Strik, W.K., Henggeler, B., Koenig, T., Koukkou, M., 1998. Brain electric microstates and momentary conscious mind states as building blocks of spontaneous thinking: I. Visual imagery and abstract thoughts. Int. J. Psychophysiol. 29, 1-11.

Marques, J.P., Kober, T., Krueger, G., van der Zwaag, W., Van de Moortele, P.F., Gruetter, R., 2010. MP2RAGE, a self bias-field corrected sequence for improved segmentation and T1-mapping at high field. Neuroimage 49, 1271-1281.

Meehan, T.P., Bressler, S.L., 2012. Neurocognitive networks: findings, models, and theory. Neurosci. Biobehav. Rev. 36, 2232-2247.

Michel, C.M., Koenig, T., 2018. EEG microstates as a tool for studying the temporal dynamics of whole-brain neuronal networks: a review. Neuroimage 15, 577-593.

Milligan, G.W., Cooper, M.C., 1985. An examination of procedures for determining the number of clusters in a data set. Psychometrika 50, 159-179.

Milz, P., Faber, P.L., Lehmann, D., Koenig, T., Kochi, K., Pascual-Marqui, R.D., 2016a. The functional significance of EEG microstates-Associations with modalities of thinking. Neuroimage 125, 643-656.

Milz, P., Pascual-Marqui, R.D., Lehmann, D., Faber, P.L., 2016b. Modalities of thinking: state and trait effects on cross-frequency functional independent brain networks. Brain Topogr. 29, 477-490.

Moscovitch, M., Cabeza, R., Winocur, G., Nadel, L., 2016. Episodic memory and beyond: the Hippocampus and neocortex in transformation. Annu. Rev. Psychol. 67, 105-134.

Murray, M.M., Brunet, D., Michel, C.M., 2008. Topographic ERP analyses: a step-by-step tutorial review. Brain Topogr. 20, 249-264.

Panda, R., Bharath, R.D., Upadhyay, N., Mangalore, S., Chennu, S., Rao, S.L., 2016. Temporal dynamics of the default mode network characterize meditation-induced alterations in consciousness. Front. Hum. Neurosci. 10, 372.
Pascual-Marqui, R.D., Michel, C.M., Lehmann, D., 1995. Segmentation of brain electrical activity into microstates: model estimation and validation. IEEE (Inst. Electr. Electron. Eng.) Trans. Biomed. Eng. 42, 658-665.

Perogamvros, L., Baird, B., Seibold, M., Riedner, B., Boly, M., Tononi, G., 2017. The phenomenal contents and neural correlates of spontaneous thoughts across wakefulness, NREM sleep, and REM sleep. J. Cogn. Neurosci. 29, 1766-1777.

Perrin, F., Pernier, J., Bertrand, O., Echallier, J.F., 1989. Spherical splines for scalp potential and current density mapping. Electroencephalogr. Clin. Neurophysiol. 72, 184-189.

Power, J.D., Cohen, A.L., Nelson, S.M., Wig, G.S., Barnes, K.A., Church, J.A., Vogel, A.C., Laumann, T.O., Miezin, F.M., Schlaggar, B.L., Petersen, S.E., 2011. Functional network organization of the human brain. Neuron 72, 665-678.

Power, J.D., Schlaggar, B.L., Petersen, S.E., 2014. Studying brain organization via spontaneous fMRI signal. Neuron 84, 681-696.

Ptak, R., Schnider, A., Fellrath, J., 2017. The dorsal frontoparietal network: a core system for emulated action. Trends Cognit. Sci. 21, 589-599.

Raichle, M.E., 2015. The brain's default mode network. Annu. Rev. Neurosci. 38, 433-447.

Rieger, K., 2016. 15 Years of microstate research in schizophrenia - where are we? A meta-analysis. Front. Psychiatry 7, 1-7.

Rubin, D.C., Schrauf, R.W., Greenberg, D.L., 2003. Belief and recollection of autobiographical memories. Mem. Cognit. 31, 887-901.

Schacter, D.L., Addis, D.R., Hassabis, D., Martin, V.C., Spreng, R.N., Szpunar, K.K., 2012. The future of memory: remembering, imagining, and the brain. Neuron 76, 677-694.

Seitzman, B.A., Abell, M., Bartley, S.C., Erickson, M.A., Bolbecker, A.R., Hetrick, W.P., 2017. Cognitive manipulation of brain electric microstates. Neuroimage 146, $533-543$.

Seth, A.K., Baars, B.J., 2005. Neural darwinism and consciousness. Conscious. Cognit. 14, $140-168$.

Setsompop, K., Gagoski, B.A., Polimeni, J.R., Witzel, T., Wedeen, V.J., Wald, L.L., 2012. Blipped-controlled aliasing in parallel imaging for simultaneous multislice echo planar imaging with reduced g-factor penalty. Magn. Reson. Med. 67, 1210-1224.

Shirer, W.R., Ryali, S., Rykhlevskaia, E., Menon, V., Greicius, M.D., 2012. Decoding subject-driven cognitive states with whole-brain connectivity patterns. Cerebr. Cortex 22, 158-165.

Simons, J.S., Peers, P.V., Mazuz, Y.S., Berryhill, M.E., Olson, I.R., 2010. Dissociation between memory accuracy and memory confidence following bilateral parietal lesions. Cerebr. Cortex 20, 479-485.

Smallwood, J., Schooler, J.W., 2015. The science of mind wandering: empirically navigating the stream of consciousness. Annu. Rev. Psychol. 66, 487-518.

Smith, S.M., 2002. Fast robust automated brain extraction. Hum. Brain Mapp. 17, 143-155.

Smith, S.M., Fox, P.T., Miller, K.L., Glahn, D.C., Fox, P.M., Mackay, C.E., Filippini, N., Watkins, K.E., Toro, R., Laird, A.R., Beckmann, C.F., 2009. Correspondence of the brain's functional architecture during activation and rest. Proc. Natl. Acad. Sci. U. S. A. $106,13040-13045$.

Spreng, R.N., Grady, C.L., 2010. Patterns of brain activity supporting autobiographical memory, prospection, and theory of mind, and their relationship to the default mode network. J. Cogn. Neurosci. 22, 1112-1123.

Spreng, R.N., Schacter, D.L., 2012. Default network modulation and large-scale network interactivity in healthy young and old adults. Cerebr. Cortex 22, 2610-2621.

Stawarczyk, D., D'Argembeau, A., 2015. Neural correlates of personal goal processing during episodic future thinking and mind-wandering: an ALE meta-analysis. Hum. Brain Mapp. 36, 2928-2947.

Steinvorth, S., Levine, B., Corkin, S., 2005. Medial temporal lobe structures are needed to re-experience remote autobiographical memories: evidence from H.M. and W.R. Neuropsychologia 43, 479-496.

Sverak, T., Albrechtova, L., Lamos, M., Rektorova, I., Ustohal, L., 2018. Intensive repetitive transcranial magnetic stimulation changes EEG microstates in schizophrenia: a pilot study. Schizophr. Res. 193, 451-452.

Svoboda, E., McKinnon, M.C., Levine, B., 2006. The functional neuroanatomy of autobiographical memory: a meta-analysis. Neuropsychologia 44, 2189-2208.

Tomescu, M.I., Rihs, T.A., Becker, R., Britz, J., Custo, A., Grouiller, F., Schneider, M., Debbane, M., Eliez, S., Michel, C.M., 2014. Deviant dynamics of EEG resting state pattern in 22q11.2 deletion syndrome adolescents: a vulnerability marker of schizophrenia? Schizophr. Res. 157, 175-181.

Tomescu, M.I., Rihs, T.A., Rochas, V., Hardmeier, M., Britz, J., Allali, G., Fuhr, P., Eliez, S., Michel, C.M., 2018. From swing to cane: sex differences of EEG resting-state temporal patterns during maturation and aging. Dev Cogn Neurosci 31, 58-66.

Tononi, G., Boly, M., Massimini, M., Koch, C., 2016. Integrated information theory: from consciousness to its physical substrate. Nat. Rev. Neurosci. 17, 450-461.

Tulving, E., 1985. Memory and consciousness. Can. Psychol. 26, 1-12.

van de Ville, D., Britz, J., Michel, C.M., 2010. EEG microstate sequences in healthy humans at rest reveal scale-free dynamics. Proc. Natl. Acad. Sci. U. S. A. 107, 18179-18184.

Vidaurre, D., Smith, S.M., Woolrich, M.W., 2017. Brain network dynamics are hierarchically organized in time. Proc. Natl. Acad. Sci. U. S. A. 114, 12827-12832.

Woletz, M., Hoffmann, A., Tik, M., Sladky, R., Lanzenberger, R., Robinson, S., Windischberger, C., 2019. Beware detrending: optimal preprocessing pipeline for low-frequency fluctuation analysis. Hum. Brain Mapp. 10, 1571-1582.

Zalesky, A., Fornito, A., Cocchi, L., Gollo, L.L., Breakspear, M., 2014. Time-resolved resting-state brain networks. Proc. Natl. Acad. Sci. U. S. A. 111, 10341-10346.

Zou, Q.H., Zhu, C.Z., Yang, Y., Zuo, X.N., Long, X.Y., Cao, Q.J., Wang, Y.F., Zang, Y.F., 2008. An improved approach to detection of amplitude of low-frequency fluctuation (ALFF) for resting-state fMRI: fractional ALFF. J. Neurosci. Methods 172, 137-141. 